\title{
SETD2 mutations in primary central nervous system tumors
}

\author{
Angela N. Viaene ${ }^{1}$, Mariarita Santi', Jason Rosenbaum², Marilyn M. Li', Lea F. Surrey ${ }^{1}$ and MacLean P. Nasrallah ${ }^{2,3^{*}}$ (D
}

\begin{abstract}
Mutations in SETD2 are found in many tumors, including central nervous system (CNS) tumors. Previous work has shown these mutations occur specifically in high grade gliomas of the cerebral hemispheres in pediatric and young adult patients. We investigated SETD2 mutations in a cohort of approximately 640 CNS tumors via next generation sequencing; 23 mutations were detected across 19 primary CNS tumors. Mutations were found in a wide variety of tumors and locations at a broad range of allele frequencies. SETD2 mutations were seen in both low and high grade gliomas as well as non-glial tumors, and occurred in patients greater than 55 years of age, in addition to pediatric and young adult patients. High grade gliomas at first occurrence demonstrated either frameshift/truncating mutations or point mutations at high allele frequencies, whereas recurrent high grade gliomas frequently harbored subclones with point mutations in SETD2 at lower allele frequencies in the setting of higher mutational burdens. Comparison with the TCGA dataset demonstrated consistent findings. Finally, immunohistochemistry showed decreased staining for H3K36me3 in our cohort of SETD2 mutant tumors compared to wildtype controls. Our data further describe the spectrum of tumors in which SETD2 mutations are found and provide a context for interpretation of these mutations in the clinical setting.
\end{abstract}

Keywords: SETD2, Histone, Brain tumor, Glioma, Epigenetics, H3K36me3

\section{Introduction}

Histone modifying enzymes regulate gene expression and play a role in numerous genomic functions through the modification of histones and non-histone proteins [20]. The disruption of normal epigenetic mechanisms secondary to mutations in histone modifying enzymes has been implicated in tumorigenesis [2] and in chemotherapeutic resistance in cancer patients [26]. Specifically, the loss of normal histone modifying enzyme activity is thought to result in alterations in chromatin configuration, disrupting cellular transcription and predisposing a cell to cancerous development [11]. In addition, multiple epigenetic therapies are in development or undergoing testing [23].

The SETD2 gene encodes SET domain-containing 2 (SETD2), a histone modifying enzyme responsible for all trimethylation of the lysine 36 residue on Histone 3

\footnotetext{
* Correspondence: maclean.nasrallah@uphs.upenn.edu

${ }^{2}$ Department of Pathology and Laboratory Medicine, University of

Pennsylvania Perelman School of Medicine, Philadelphia, PA, USA

${ }^{3}$ Hospital of the University of Pennsylvania, FO6.089 3400 Spruce St,

Philadelphia, PA 19104, USA

Full list of author information is available at the end of the article
}

(H3K36me3) in humans. Decreases in H3K36me3 lead to alterations in gene regulation, increased spontaneous mutation frequency and chromosomal instability [13, 14]. Prior studies have indicated that loss of one allele of SETD2 does not significantly decrease levels of H3K36me3 $[5,8]$; however, it is important to note that biallelic inactivation of SETD2 may not be the sole mechanism leading to the loss of H3K36me3. For example, overexpression of other proteins such as HOX Transcript Antisense RNA (HOTAIR) can decrease levels of H3K63me3 as well [14].

SETD2-inactivating mutations have been implicated in a number of tumor types (for a review, see [14]). Most frequently, SETD2 mutations are seen in clear cell renal cell carcinoma (CCRCC) and are thought to confer a poor prognosis [16]. SETD2 mutations have also been reported in neoplasms of the central nervous system (CNS) $[1,6,27]$. These mutations have been found to be specific to pediatric and young adult high grade gliomas located in the cerebral hemispheres, affecting $15 \%$ and $8 \%$ of pediatric and adult high grade gliomas, respectively, and not found in other gliomas [6]. In the 2016 WHO Classification of Tumors of the Central Nervous

(c) The Author(s). 2018 Open Access This article is distributed under the terms of the Creative Commons Attribution 4.0 International License (http://creativecommons.org/licenses/by/4.0/), which permits unrestricted use, distribution, and 
System, SETD2 mutations are listed under frequent genetic alterations in pediatric (but not adult) high-grade diffuse astrocytic tumors within the cerebral hemispheres [18].

Western blot studies have shown SETD2-mutant gliomas have decreased levels of H3K36me3, indicating that the mutations in these tumors are loss-of-function [6]. Similarly, immunohistochemical studies of CCRCC, chondroblastomas, and chordomas have been used to demonstrate decreased staining for H3K36me3 in tumors with SETD2 mutations $[8,16,19,25]$. To our knowledge, immunohistochemistry has not been used to evaluate levels of H3K36me3 in SETD2-mutant brain tumors.

Here we describe 19 cases of CNS tumors with mutations in SETD2. SETD2 mutation allele frequency and co-occurring mutations in other genes are investigated, and results are correlated with the effects of SETD2 mutations on epigenetic change, specifically histone methylation and acetylation as shown by immunohistochemistry for H3K36me3, H3K36ac and H3K27me3. Our findings indicate that SETD2 mutations occur at a wide range of allele frequencies in a variety of tumors of the central nervous system and that those mutations most likely to have functional impact on the gene product are seen most often but not exclusively in pediatric and young adult high grade gliomas of the cerebral hemispheres.

\section{Materials and methods}

This study was approved by an independent institutional review board at the Hospital of the University of Pennsylvania (HUP IRB 827290). All CNS tumors with SETD2 mutations identified on routine next generation sequencing (NGS) studies performed September 17, 2016 through June 30, 2017 at HUP and from February 1, 2016 to June 30, 2018 at CHOP are included in the current study. Fifteen tumors from HUP and four tumors from Children's Hospital of Philadelphia (CHOP) are included. Patients whose tumor showed single nucleotide polymorphisms or otherwise benign variants in SETD2 were excluded.

\section{Next generation sequencing}

At our institutions, targeted NGS of brain tumor specimens is performed as part of routine patient care. Genomic tumor testing was performed at the Center for Personalized Diagnostics (CPD) at the University of Pennsylvania and the Division of Genomic Diagnostics (DGD) at the CHOP, both CLIA-approved laboratories. Genomic DNA from brain tumor specimens is extracted from fresh tissue, formalin-fixed paraffin-embedded tissues or frozen tissue. Tumor DNA is sequenced on an Illumina MiSeq or HiSeq, and the data are analyzed using in-house bioinformatics pipelines.

At the CPD, the Solid Tumor Sequencing panel uses a custom Agilent HaloPlex library preparation (Agilent,
Santa Clara, CA) to cover approximately 0.5 megabases, including the entire exonic (coding) sequence of 152 genes, +10 base pairs of intronic sequence. The 152 genes sequenced on the CPD panel may be found at (https://www.pennmedicine.org/departments-and-centers/ center-for-personalized-diagnostics/gene-panels). The library preparation includes unique molecular identifiers to identify duplicate reads. Specimens are sequenced on the Illumina HiSeq 2500 platform (Illumina, San Diego, CA) using multiplexed, paired end reads. Analysis and interpretation is performed using a customized bioinformatics pipeline, Halo_v1.2. All variants are annotated with reference to the hg19 Genome build. Variants are reported according to HGVS nomenclature and classified into 3 categories: Disease-Associated Variants, Variants of Uncertain Significance, and Benign. Variant allele frequency (VAF) is defined as the number of reads of a variant from the reference sequence divided by the total number of reads at that base.

The Comprehensive Solid Tumor Panel v1 at CHOP includes sequence and copy number analyses of 237 cancer genes, and 586 known fusions and many more novel fusions associated with 106 fusion gene partners. The genes included in the panel can be found at (https:// www.testmenu.com/chop/Tests/785967). Fusion genes were evaluated by targeted RNA-seq using anchored multiplex PCR with custom designed primers (ArcherDx, Boulder, CO). Full exonic and select intronic/promotor sequence of 237 cancer genes were evaluated by next generation sequencing. Regions of interest were captured using SureSelect ${ }^{\mathrm{QTX}}$ target enrichment technology (Agilent Technologies, Santa Clara, CA). Sequencing was performed on Illumina MiSeq or HiSeq (San Diego, CA). Sequencing data were processed using the homebrew software ConcordS v1 and NextGENe v2 NGS Analysis Software (Softgenetics, State College, PA). Variant interpretation was performed according to AMP/ASCO/CAP standards and guidelines for somatic variant interpretation and reporting [15].

\section{Immunohistochemistry}

All tumors with nonsense or frameshift mutations in SETD2 and those with missense mutations with AF greater than $40 \%$ were used for immunohistochemical (IHC) studies. In addition, glioblastomas, anaplastic astrocytomas, pilocytic astrocytomas and meningiomas confirmed to be SETD2-wildtype by NGS were used as controls.

H3K36me3 (Abcam ab9050), H3K36ac (Abcam ab177179), and H3K27me3 (Cell Signaling 9733) antibodies were used to stain formalin fixed paraffin embedded slides. Staining was performed on a Bond Max automated staining system (Leica Biosystems). The Bond Refine polymer staining kit (Leica Biosystems) was used. The standard protocol was followed with the exception 
of the primary antibody incubation which was extended to $1 \mathrm{~h}$ at room temperature. The antibodies were used at 1:5 K (H3K36me3), 1:100 (H3K36ac) and 1:150 (H3K36me3) dilutions and antigen retrieval was performed with E1 (H3K36me3 \& H3K36ac) or E2 (H3K27me3) (Leica Biosystems) retrieval solution for $20 \mathrm{~min}$. Slides were rinsed, dehydrated through a series of ascending concentrations of ethanol and xylene, then coverslipped.

Immunohistochemistry was scored using the semiquantitative $\mathrm{H}$-score system. $\mathrm{H}$-scores were calculated as $3 \mathrm{x}$ the percentage of strongly staining nuclei $+2 x$ the percentage of moderately staining nuclei + the percentage of weakly staining nuclei (giving a range of 0 to 300). The $\mathrm{H}$-scores were independently assessed by two neuropathologists board-certified by the American Board of Pathology (MPN and ANV) on de-identified slides.

\section{TCGA}

The Cancer Genome Atlas (TCGA) dataset was retrieved from cbioportal (http://www.cbioportal.org) by searching for SETD2 mutations in "CNS/Brain" tumors (headings: diffuse glioma, glioblastoma, oligodendroglioma, pilocytic astrocytoma and medulloblastoma). The search was performed 11/30/2017.

\section{Statistical analysis}

Statistical analysis was performed using SPSS version 23.0 (IBM Corp.). Statistical significance was defined as $p<.05$ and based on two-tailed tests. For the analysis of the number of co-occurring mutations in SETD2-mutant tumors, only data from the CPD were used in calculations.

\section{Results}

From September 17, 2016 through June 30, 2017, approximately 400 CNS tumors, including metastases, were sequenced at the University of Pennsylvania CPD. From February 1, 2016 to June 30, 2018 approximately 240 CNS tumors were sequenced at the DGD at CHOP. Nineteen primary brain tumors (fifteen at the University of Pennsylvania and four at CHOP) with SETD2 mutations were identified on routine NGS studies (Tables 1 and 2). Eleven tumors had nonsense or frameshift mutations (truncating mutations) in SETD2 and eight had missense mutations. The age of the patients ranged from 9 to 80 years old (mean 43 years, median 42 years), and there was no statistically significant difference $(p=0.49)$ in age between patients with nonsense or frameshift mutations and those with missense mutations (Fig. 1a); however, of high grade gliomas, recurrences often showed missense mutations, whereas frameshift and nonsense mutations were preferentially seen in de novo tumors (Tables 1 and 2). The male to female ratio of the cohort was 3:1.
Twelve of these SETD2-mutant tumors were located within the cerebral hemispheres while seven occurred outside the hemispheres (two extra-axial, one thalamic, and four posterior fossa; Fig. 1b). A broad range of tumor histologies were seen, including high grade gliomas ( $n=10,62.5 \%$, with 4 of them recurrent), low grade astrocytic tumors $(n=5,12.5 \%)$, atypical meningiomas $(n=2,12.5 \%)$, a medulloblastoma $(n=1,6.3 \%)$ and a choroid plexus papilloma $(n=1,6.3 \%)$. Examples of tumor histology are show in Fig. 1d. Overall, eleven of the SETD2-mutant tumors were classified as high grade (WHO grade III or IV) and eight were low grade tumors (WHO grade I or II) (Tables 1 and 2).

In total, 23 SETD2 changes amongst the 19 tumors were detected at a wide range of VAF (range 2-51\%); 4 tumors had more than one SETD2 missense mutation, 3 of which were recurrent high grade gliomas, and the fourth a medulloblastoma. No statistically significant difference $(p=0.49)$ in VAF was seen between truncating mutations and missense mutations.

The detected mutations were distributed throughout SETD2 with the majority of the high grade glioma nonsense or frameshift mutations occurring $5^{\prime}$ to the SET domain (VAF 4-44\%) (Fig. 2a). The nonsense or frameshift mutations for the low grade gliomas occurred throughout the SETD2 gene (VAF 6-34\%).

Missense mutations occurred throughout the SETD2 gene, and in gliomas, were found predominantly in recurrent high grade gliomas, including recurrent glioblastomas (Table 2). The exceptions were patients 10 and 12. For patient 10 in Table 2, diagnosed with an $I D H$-wildtype anaplastic astrocytoma, a p.I1398T change in SETD2 was found; however, this may represent a benign single nucleotide polymorphism as it is seen at $>0.1 \%$ frequency in the Ashkenazi Jewish population (http://gnomad.broadinstitute.org/) [12]. Patient 12, with otherwise similar characteristics, showed SETD2 p.A2242V, which we classify as a variant of uncertain significance, given that is not been identified previously. The remaining missense mutations found in SETD2 may represent changes found with the increased mutational load seen with tumor recurrence in this cohort $(4.57 \pm 3.40$ mutations in recurrent tumors vs. $1.41 \pm 1.24$ mutations in primary tumors, $p<0.01$ ), and known in the literature, particularly after treatment with temozolomide [3, 9]. The largest number of co-occurring mutations (11) was seen in patient 14 following chemotherapy and radiation. Of note, this patient also had two MM in SETD2. Within the adult cohort, tumors with missense mutations in SETD2 had more concurrent mutations than did those with truncations of SETD2 (5.17 \pm 3.31 vs. $1.50 \pm 1.35, p<0.05)$. Mutations in EGFR were found to be the most commonly co-occurring change with SETD2 changes and were seen in $40 \%$ of the high grade gliomas in this cohort, similar to 


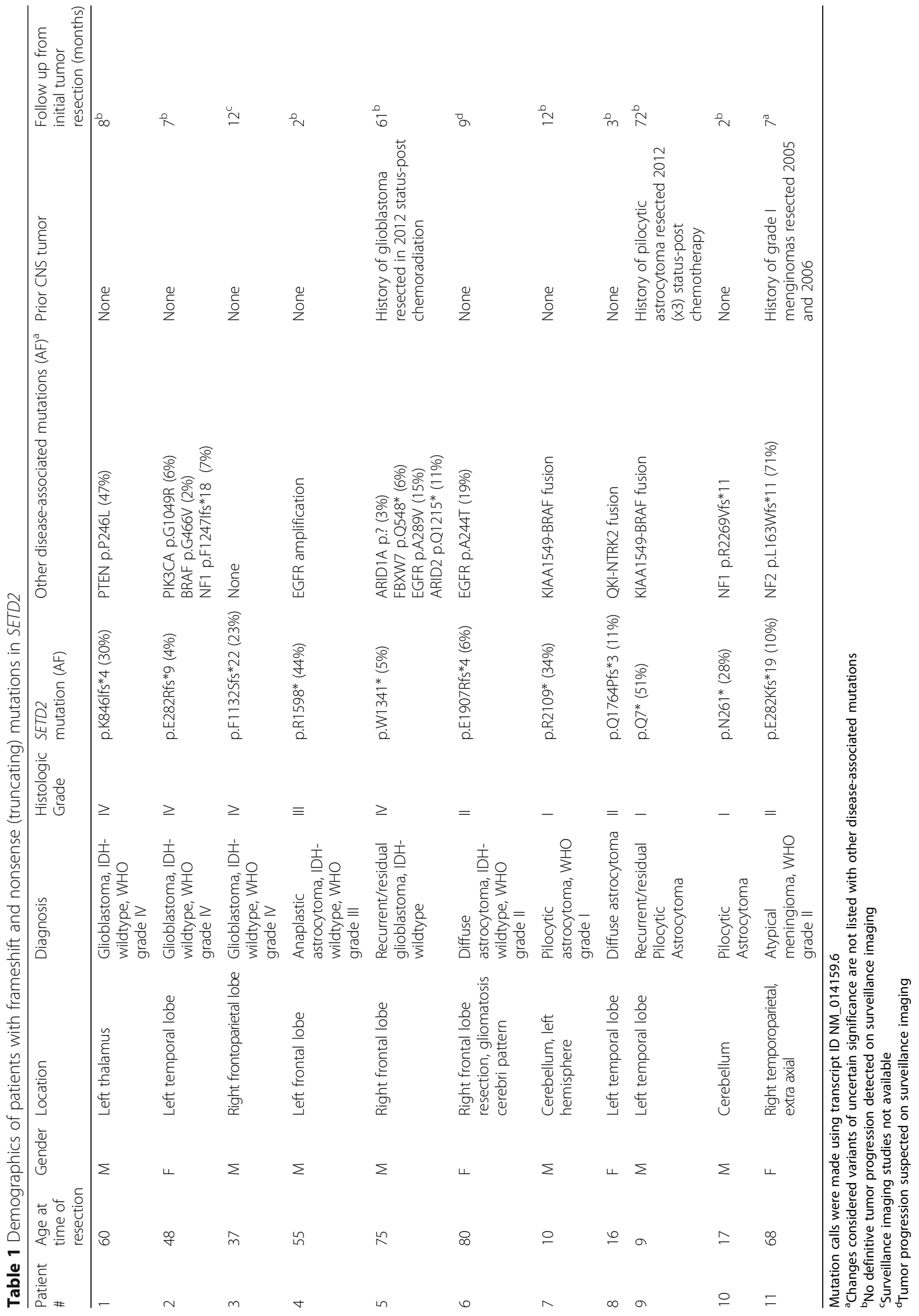




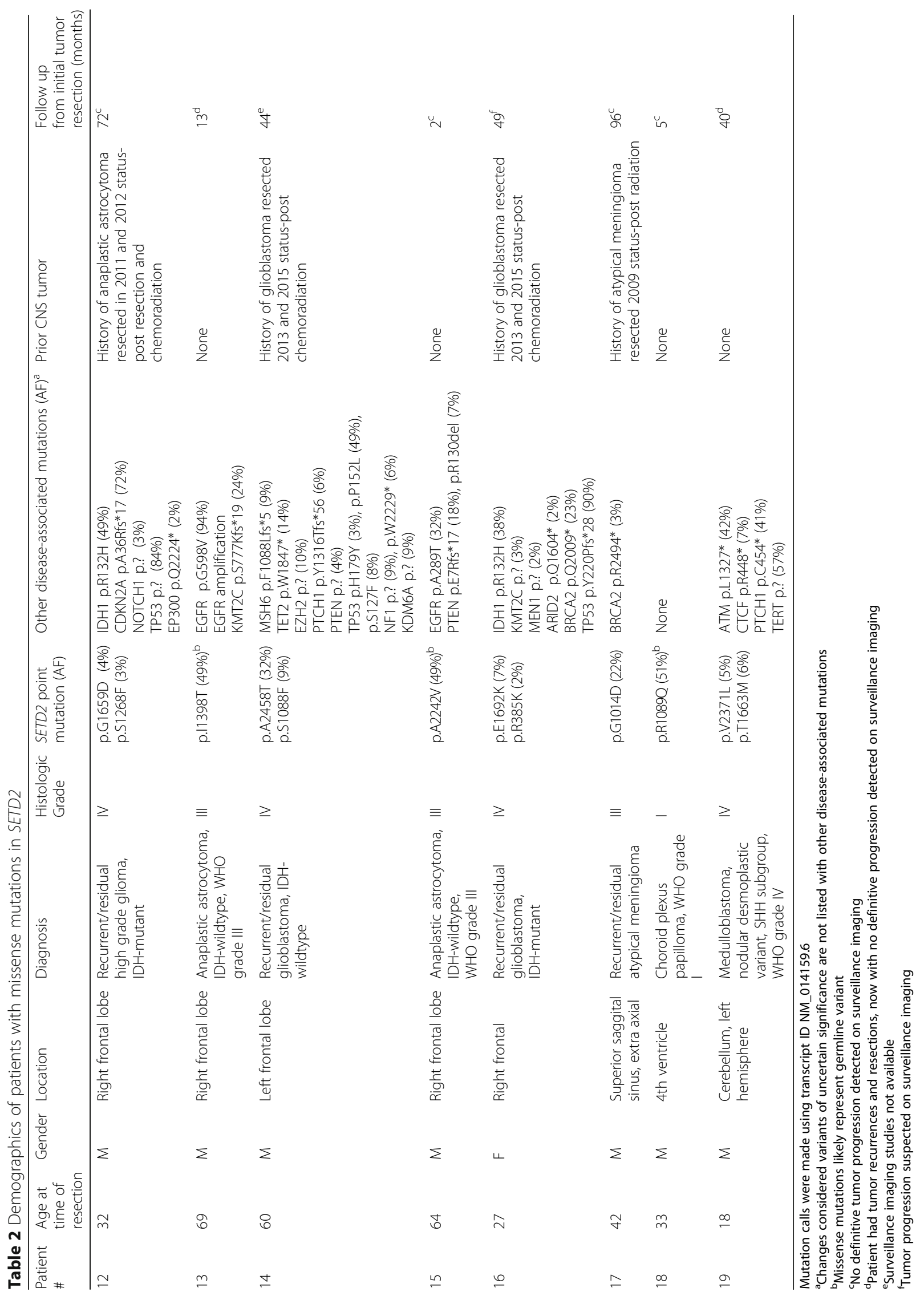



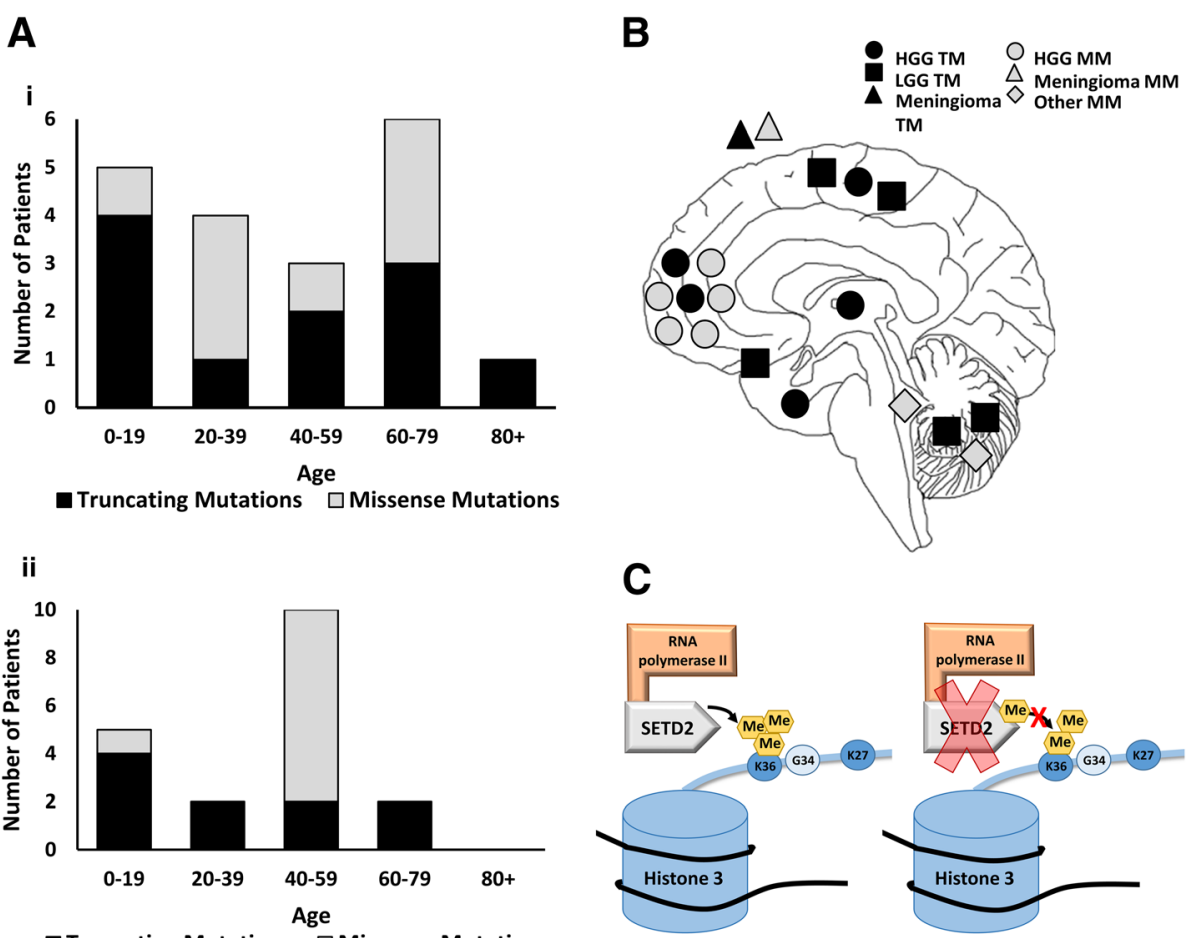

C

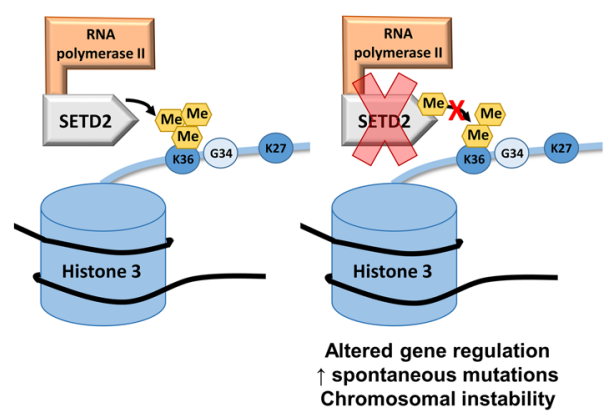

D

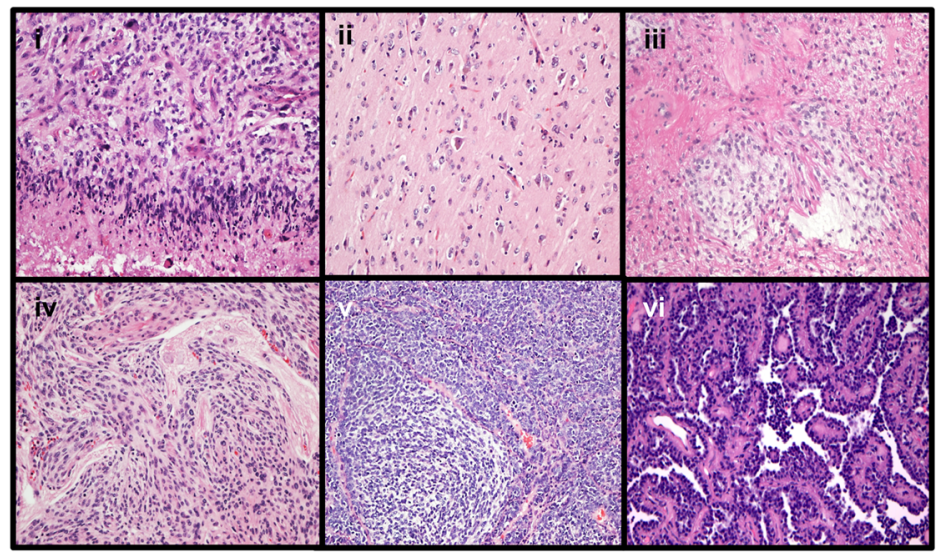

Fig. 1 Demographics, locations and histologies of SETD2 mutant brain tumors. a Histograms of patient ages at time of tumor resection for the 19 cases presented in the current study (i) and from the TCGA database (ii). $\mathbf{b}$ Schematic representation of SETD2 mutant tumor locations within the CNS. c A schematic illustrating the proposed epigenetic effects of SETD2 alterations. d Representative histologies of SETD2 mutant tumors: Glioblastoma (i), Diffuse astrocytoma (ii), Pilocytic astrocytoma (iii), Atypical meningioma (iv), Medulloblastoma (v), Choroid plexus papilloma (vi). All tumors stained with Hematoxylin and Eosin. All photographs taken at 200x magnification. Truncating mutations (TM), missense mutation (MM)

the frequency of EGFR mutations found in high grade gliomas overall (Fig. 2b) [1]. Pathogenic mutations in TP53 were seen in $30 \%$ of high grade gliomas with SETD2 changes, and IDH mutations were seen in $20 \%$. However, TP53 and IDH mutations were only seen in tumors with missense mutations in SETD2 and not those with nonsense or frameshift mutations in SETD2. No mutations in H3F3A were seen to co-occur with SETD2 changes.

\section{Immunohistochemistry}

Immunohistochemistry for H3K36me3, H3K36ac and H3K27me3 expression were assessed with $\mathrm{H}$-scores by two neuropathologists (ANV and MPN) on SETD2-mutant tumors and histologic controls confirmed to be wildtype for SETD2 by NGS. Gliomas with SETD2 mutations showed significantly lower $\mathrm{H}$-scores for H3K36me3 compared to wildtype controls (140.8 \pm 65.4 

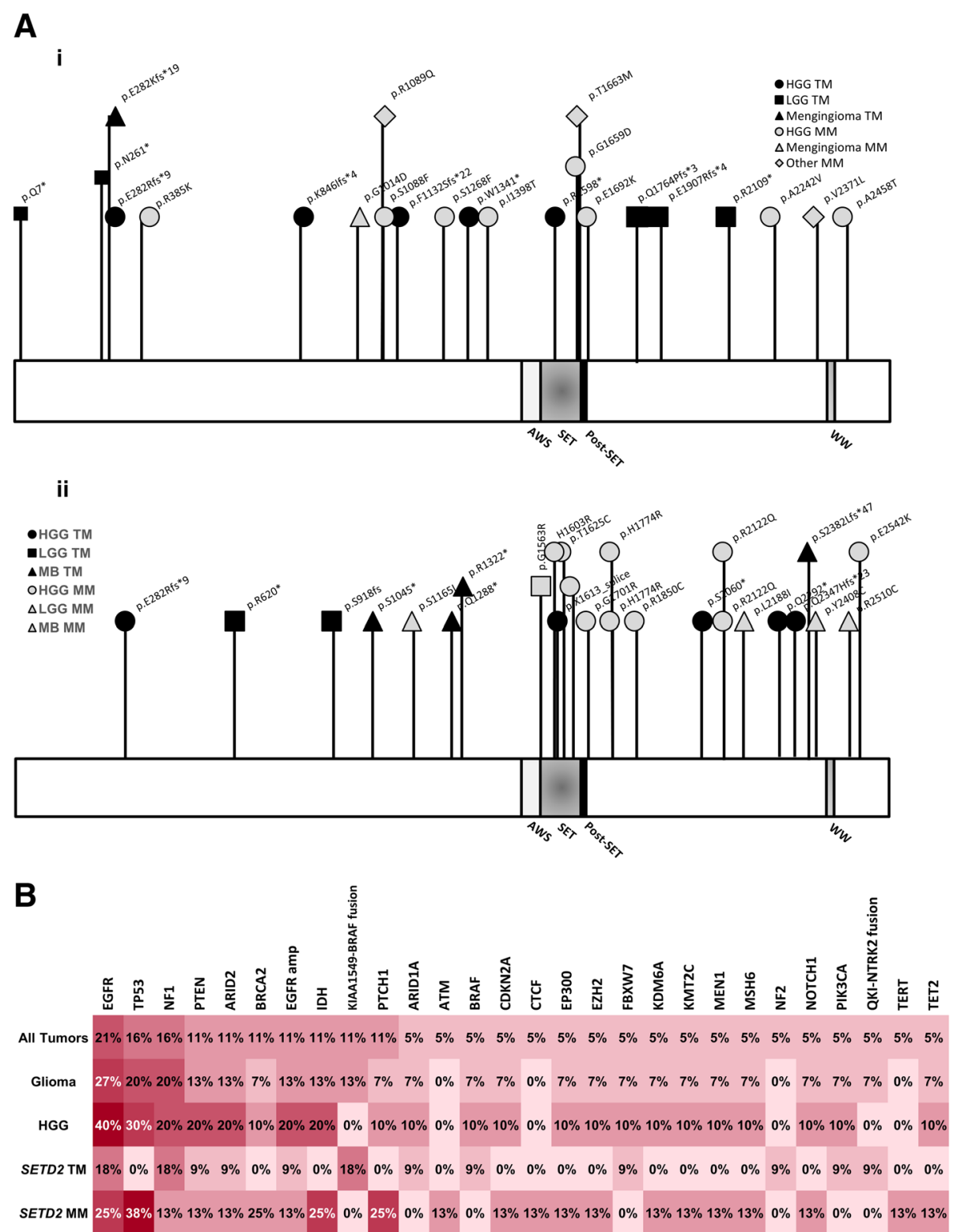

Fig. 2 Location of mutations within SETD2 and co-occurring pathogenic mutations. a Schematic representation of the locations of mutations in SETD2 for the 16 cases presented in the current study (i) and the TCGA database (ii) (AWS, Associated with SET). b Other pathogenic mutations co-occurring in tumors with SETD2 mutations with the percentage of tumors with each mutation is labeled within each cell. Darker shading corresponds to higher percentages. Truncating mutations (TM), missense mutation (MM)

vs. $228.8 \pm 29.1, p<0.01$ ) (Fig. 3). In contrast, statistically significant differences in staining for H3K36ac and H3K27me3 between SETD2 mutants and controls were not observed (H3K36ac: $140.1 \pm 35.7$ vs. $160.0 \pm 41.8$, $p=0.13$; H3K27me3: $206.1 \pm 24.2 \mathrm{vs.} 190.8 \pm 33.5, p=0.23$ ). There was no correlation between $\mathrm{AF}$ and $\mathrm{H}$-score for any of the immunohistochemical markers [H3K36me3: $F_{1,8}=0.55, p=.48$ with an $\mathrm{R}^{2}$ of 0.06 (Fig. 3c); H3K36ac: $F_{1,8}=0.54, p=.48$ with an $\mathrm{R}^{2}$ of 0.06 ; H3K27me3: $F_{1,8}=0.46, p=.53$ with an $\mathrm{R}^{2}$ of 0.08]. The concordance correlation coefficients for the three antibodies were as follows: H3K36me3 concordance correlation coefficient of 0.88 [95\% CI 0.69-0.96]; H3K36ac concordance correlation coefficient of 0.58 (95\% CI 0.12-0.83); H3K27me3: concordance correlation coefficient of 0.51 (95\% CI 0.16-0.75).

\section{TCGA}

The results shown here are based upon data generated by the TCGA Research Network: http://cancergenome. nih.gov/, via http://www.cbioportal.org/ [4, 7]. A total of 22 CNS tumors with SETD2 mutations were identified across the cohorts included in the TCGA datasets, with 

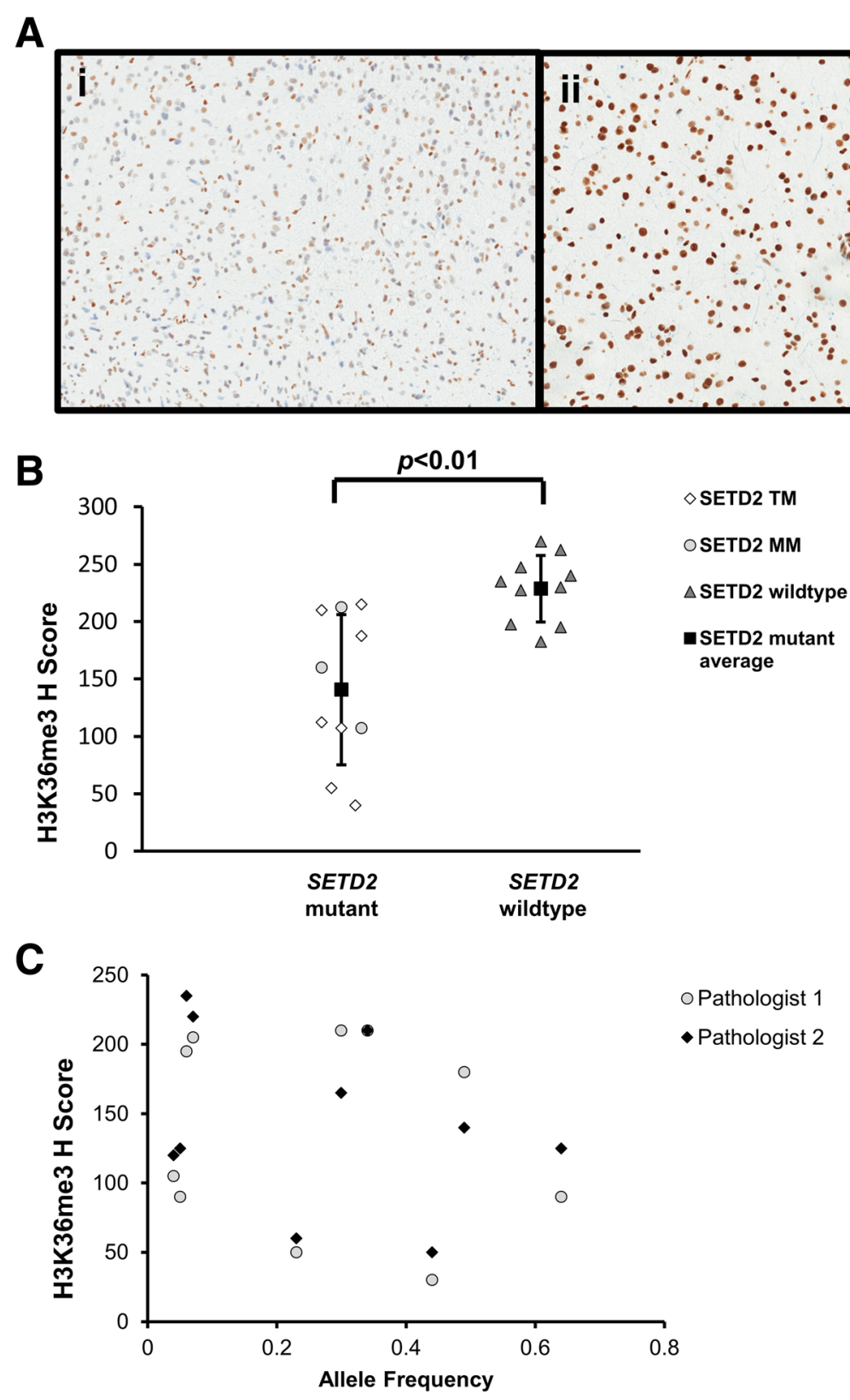

Fig. 3 Immunohistochemical staining for H3K36me3. a Examples of immunohistochemical staining for H3K36me3 in high grade gliomas with a truncating mutation in SETD2 (i) and wildtype control (ii); both images were taken at 200x magnification. $\mathbf{b} \mathrm{H}$ scores for SETD2 mutant tumors and wildtype tumors. Averages for each group are shown as black squares and with error bars representing the standard deviation. $\mathbf{c} \mathrm{H}$ scores calculated by two independent pathologists for SETD2 mutants plotted against allele frequency. Truncating mutations (TM), missense mutation (MM)

0-14\% of CNS tumors harboring a SETD2 mutation depending on the cohort (Fig. 4). Eleven tumors had truncating mutations, ten had missense mutations and one had a splice-site mutation. The age of the patients ranged from 2 to 74 years (mean $40.1 \pm 23.6$ years) with a male to female ratio of 2.3:1. There was no statistically significant difference $(p=0.22)$ in age between patients with truncating mutations and those with missense mutations. SETD2 mutations were found in high grade gliomas $(n=14,63 \%)$, low grade gliomas $(n=3,14 \%)$, and medulloblastomas $(n=5,23 \%)$. The low grade gliomas included two pilocytic astrocytomas and an IDH-mutant, $1 \mathrm{p} / 19 \mathrm{q}$-codeleted, WHO grade II oligodendroglioma. Data on the location of the tumors is limited; a study of glioblastomas found that all tumors with SETD2 mutations were located in the cerebral hemispheres [6]. However, it is likely SETD2 mutant tumors were also present in the posterior fossa as mutations 

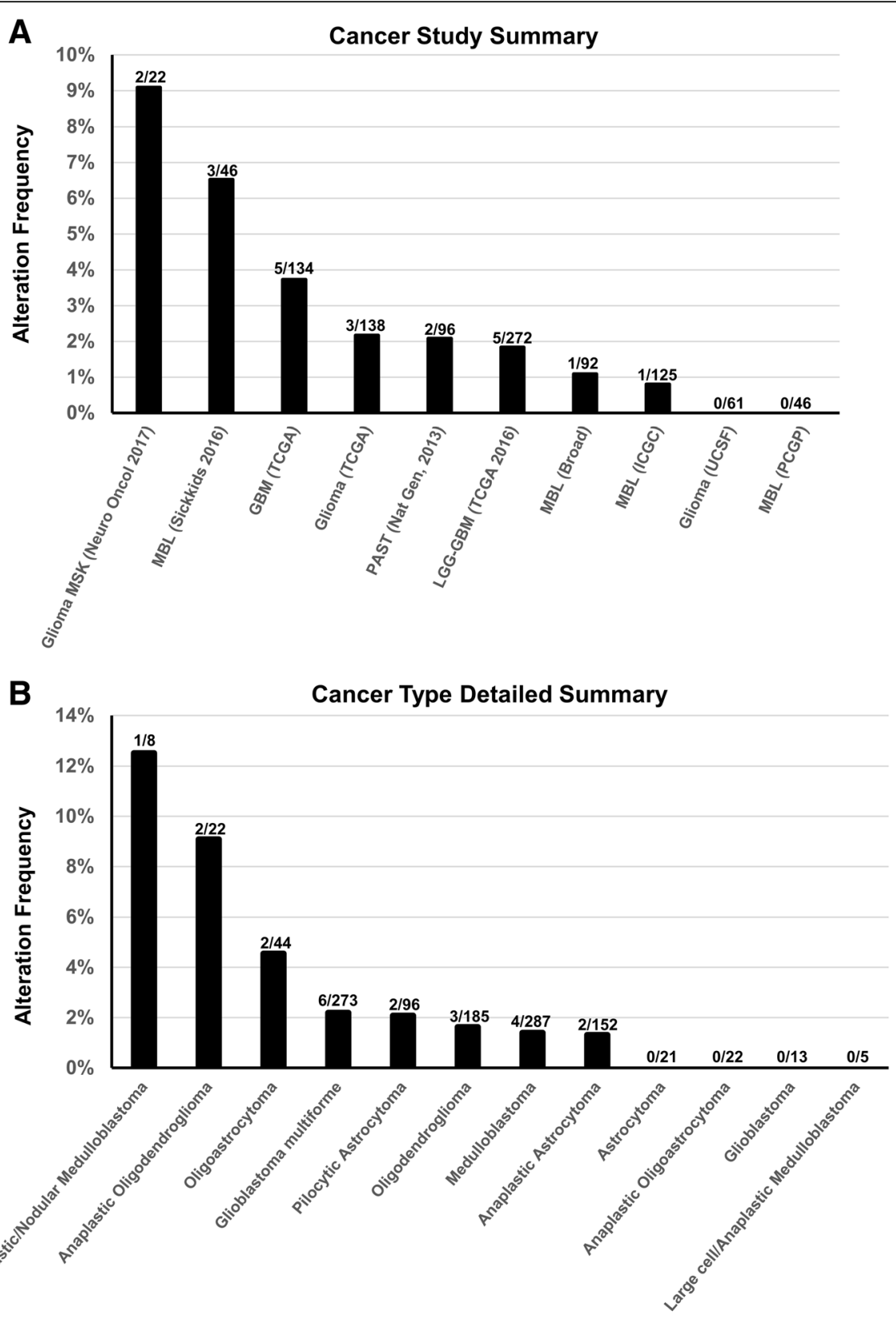

Fig. 4 SETD2 mutations in CNS tumors retrieved from the TCGA database. a Frequency of SETD2 alterations detected per study. b Frequency of SETD2 alterations detected per tumor type. The number of cases with SETD2 variants over the denominator of the total number of analyzed cases for each group is indicated above the bars

were seen in 5 medulloblastomas and 2 pilocytic astrocytomas, tumors which both have a strong association with the posterior fossa. In total, 26 SETD2 mutations were seen among the 22 tumors with one medulloblastoma having 3 SETD2 missense mutations. For the 11 tumors for which data on AF was available, the frequency ranged from 5 to $48 \%$. No statistically significant difference in $\mathrm{AF}$ was seen between truncating mutations and missense mutations $(p=0.82)$. The mutations were distributed throughout SETD2 (Fig. 2 aii). Survival data is available on 16 patients (15 patients with gliomas and 1 patient with medulloblastoma). The average follow-up was $19.1 \pm 17.3$ months (range 5 to 72 months) for all tumors and $15.8 \pm 11.4$ months (range 5 to 45 months) for patients with high grade gliomas. Twelve patients were still living. High grade gliomas with TM had an average follow-up of $13.2 \pm 10.8$ months and those with MM had an average follow-up of $17.7 \pm 12.3$ months $(p=.52)$. Four deceased patients all had high grade gliomas (two of which were recurrent) with an average 
survival of $16.3 \pm 10.0$ months (range 7 to 30 months). Two of the deceased patients had TM (survival 7 months and 30 months) and two patients had MM (survivals of 11 and 17 months).

\section{Discussion}

Epigenetic changes, such as DNA methylation and histone modifications affect the structure of chromatin, and therefore have a potentially broad impact on transcription. Epigenetic changes have been detected in a wide range of tumors, and a variety of drugs have been developed to target these changes; for a review, see [23]. Epigenetic mutations in gliomas include H3 K27 M mutations in diffuse midline gliomas and H3.3 G34R/V mutations in gliomas of the cerebral hemispheres [18]. In diffuse midline gliomas, the H3 K27 M mutation has been shown to globally reduce levels of H3K27me3 levels, altering transcription and driving tumorigenesis. Several therapies that target enzymes responsible for chromatin modifications in these gliomas have been developed; for a review, see [17]. In gliomas, mutations have also been reported in SETD2, whose protein product is an enzyme responsible for trimethylation of the lysine 36 residue on Histone 3 (H3K36me3) in humans. These mutations potentially decrease H3K36me3, which may alter gene regulation, increase spontaneous mutation frequency and lead to chromosomal instability, with theoretically targetable effects (Fig. 1c).

Our investigation of SETD2 mutations in brain tumors yields findings consistent with the previous report of SETD2 mutations in gliomas, which shows that loss-of-function SETD2 mutations occur in older children and young adults in high grade gliomas of the cerebral cortex [6]. In addition, we expand on those results to demonstrate that subclonal mutations in SETD2 are seen in a broad range of tumors and locations. Prior studies focusing on gliomas illustrate that SETD2 mutations are found nearly exclusively within the cerebral hemispheres $[1,6,22,27]$. Similarly, in our cohort, mutations were most commonly seen in high grade gliomas within the cerebral hemispheres (12 of 19 tumors). However, our results include a broader range of CNS tumor types, and demonstrate SETD2 truncating mutations in atypical meningiomas and pilocytic astrocytomas, as well as missense mutations in a choroid plexus papilloma and a medulloblastoma. Data from the TCGA database also showed SETD2 mutations in pilocytic astrocytomas, an oligodendroglioma, and medulloblastomas. Prior studies of medulloblastomas did not detect mutations in SETD2 [10] suggesting SETD2 mutations are rare in these tumors. The medulloblastoma described in our cohort belongs to the Sonic Hedgehog genetic group, and has two point mutations in SETD2 with reported VAFs of 5\% and 6\%.

In contrast to previous work showing that SETD2 mutations are seen in high grade gliomas but not low grade gliomas, we found frameshift mutations in SETD2 in two diffuse astrocytomas, WHO grade II. However, one SETD2-mutant astrocytoma in an older patient (\#6) also harbors an EGFR mutation and lacks $I D H$ changes, and so is in essence a "molecular glioblastoma," in addition to radiologically showing a gliomatosis cerebri pattern of growth. Given the previous findings that SETD2 mutations are specific to high grade gliomas, the SETD2 change in this tumor may be hypothesized to indicate or correlate with aggressive behavior. The patient experienced slow radiological progression and clinical decline over 8 months despite temozolomide therapy, and subsequently transitioned to hospice and comfort care. Along the same lines, we found truncating mutations in three pilocytic astrocytomas. Although pilocytic astrocytomas are grade I tumors, they can show anaplastic changes, and one of these three tumors did have increased mitotic activity (\#7). Additionally, another pilocytic astrocytoma was a recurrent tumor with a history of three prior resections and chemotherapy (\#9).

In addition, not all patients conform to the previously reported age and tumor location profile. For example, the patient diagnosed with the diffuse astrocytoma mentioned above was 80 years old at the time of diagnosis. Also, a 60-year-old patient presented with a thalamic glioblastoma, which demonstrated a frameshift mutation in SETD2 at a 30\% VAF. Data from the TCGA database also showed a wide range of patient ages (2-74 years).

In our cohort, mutations were seen in a variety of regions within the SETD2 gene and at a broad range of VAF in tumors. In high grade gliomas, nonsense and frameshift mutations were mostly located $5^{\prime}$ to the SET domain. These findings are similar to what has been reported [6]. In contrast, in the low grade astrocytic tumors, nonsense or frameshift mutations often occurred $3^{\prime}$ to the SET domain, including in tumor \#6. Missense mutations were found throughout SETD2. The significance of the location of mutation with respect to nonsense mediated decay of the RNA is unknown. SETD2 mutations with low VAF (defined as VAF $<10 \%$ ), were seen to co-occur with an average of $3.8 \pm 1.7$ other mutations (range 1-6 mutations). Those tumors with higher SETD2 mutation VAF $(\geq 10 \%)$ had an average of $1.8 \pm 2.9$ additional co-occurring mutations (range $0-11$ mutations).

Several tumors in our cohort were recurrent/residual gliomas. Sequencing for SETD2 mutations was not performed on the prior resection specimens. However, one patient (\#13) had tumor recurrence and a subsequent resection which showed the same SETD2 mutation (p.I1398T) at a similar VAF. Patients 13, 15, and 18 had MM occurring at VAF around $50 \%$. It is possible that these mutations are germline though this cannot be confirmed as paired normal sequencing for SETD2 was not performed. 
We attempted to determine whether the SETD2 mutation resulted in a functional effect through immunohistochemical studies of epigenetic markers. If SETD2 mutations are indeed driving tumorigenesis in some CNS tumors, the exact mechanism by which this occurs also requires further elucidation. One of the leading hypotheses suggests that loss of SETD2 function in tumor cells decreases levels of H3K36me3, which subsequently leads to alterations in gene regulation, increased spontaneous mutation frequency and chromosomal instability (Fig. 1c) $[13,14]$. Evidence also indicates that increased levels of H3K36ac are seen when the levels of H3K36me3 decrease [21]. We employed IHC for H3K36me3, H3K36ac and H3K27me3 to investigate the impact of SETD2 mutations on histone methylation and acetylation. We hypothesized that decreased H3K36me3 staining and increased staining for H3К36аc would be present in SETD2 mutant tumors with mutation seen at high VAF. Additionally, prior investigations have shown that cells depleted for all H3K36-directed methyltransferases have a reduction in $\mathrm{H} 3 \mathrm{~K} 36 \mathrm{me} 3$ and also have elevated levels of H3K27me3 [19]. Based on these findings, we investigated whether staining for H3K27me3 is increased in SETD2 mutant tumors.

Immunohistochemical staining for H3K36me3 showed a statistically significant decrease in staining for SETD2 mutant gliomas compared to SETD2 wildtype histologic controls. However, given the variability in staining and the lack of correlation with allele frequency, IHC for the detection of SETD2 mutations is impractical in a clinical setting. Prior work in gliomas also found a significant decrease in levels of H3K36me3 in gliomas with heterozygous mutations in SETD2 by Western Blot [6]. In contrast, studies have indicated that bi-allelic loss of SETD2 is needed to significantly decrease levels of H3K36me3 in in vitro models and renal cell carcinoma [5, 8, 14]. For example, the most significant decreases in staining for H3K36me3 in non-CNS tumors with SETD2 mutations were seen when both SETD2 allelic copies were lost $[8,16,19,25]$. Specifically, studies of clear cell renal cell carcinoma indicate that mutations occurring at higher AFs may not result in significant decreases in H3K36me3 unless both SETD2 alleles are affected [5, 8]. Further studies are needed to assess whether this statistically significant decrease in levels of H3K36me3 in gliomas with heterozygous mutations in SETD2 has a function impact on tumorigenesis, as well as to determine if there is loss of the alternate allele.

Immunohistochemical stains for H3K36ac and H3K27me3 did not show statistically significant differences in staining between mutants and controls, although an increase in staining for $\mathrm{H} 3 \mathrm{~K} 27 \mathrm{me} 3$ were seen in SETD2 mutants compared to controls. There was no correlation between VAF and levels of staining for any antibodies though this may be due to the small number of tumors evaluated.

An alternative mechanistic hypothesis is that SETD2 mutations are interacting with other mutations to drive tumorigenesis. For example, SETD2 may bind to p53 and regulate the transcription of specific genes [28]. Studies of lung adenocarcinomas found loss of H3K36me3 lead to accelerated progression of early- and late-stage tumors; however SETD2 loss alone was not sufficient to overcome the p53-regulated barrier that suppresses the formation of higher grade adenocarcinomas [24]. It is possible that SETD2 mutations work in conjunction with other mutations such as TP53 or mutations in growth factor pathways to promote tumorigenesis. Although one of the most frequently observed concurrently mutated genes in our cohort of high grade gliomas is TP53, TP53 mutations were only seen in tumors with SETD2 missense mutations and not in those tumors with SETD2 nonsense or frameshift mutations. Most often, the co-occurrence of SETD2 and TP53 mutations was seen in recurrent gliomas, and the VAFs varied. These findings do not lend support to the hypothesis that SETD2 mutations synergize with TP53 mutations, and further studies are necessary.

In addition to TP53, the other most frequently observed genes showing mutations concurrent with SETD2 mutation within the high grade glioma subset were EGFR and PTEN, likely due to the frequency of mutation in these genes in glioblastoma. Recurrent tumors with SETD2 mutations had significantly more concurrent mutations than did first occurrence SETD2-mutant tumors. IDH mutations were present in a subset (18\%) of diffuse gliomas with SETD2 changes, which in this study were all SETD2 missense mutations rather than nonsense or frameshift mutations. Concurrent mutations in SETD2 and H3F3A were not seen. Both of these findings are consistent with prior investigation [6].

Data on the long-term survival for patients with SETD2-mutant CNS tumors is limited. Twelve of sixteen patients from the TCGA cohort were still living, and the data on follow-up does not allow any conclusions to be drawn regarding the impact of the SETD2 mutations. At the conclusion of the current study, all patients were still living. Focusing on patients with high grade gliomas, the average follow-up period from initial presentation was $18.0 \pm 24.3$ months (range 2 to 61 months) for truncating mutations and $36.0 \pm 28.3$ months (range 2 to 72 months) for missense mutations. Two tumors with SETD2 missense mutations were positive for IDH1 mutations; when comparing tumors with SETD2 missense mutations, IDH-mutant tumors had follow-up periods of 79 and 42 months versus follow-up periods ranging from 2 to 44 months with an average follow-up of $19.7 \pm 21.8$ months for $I D H$-wildtype gliomas. On average, longer follow-up data was available for patients 
with tumors with missense mutations as several of these were identified in recurrences ( 4 of 5 high grade gliomas with missense mutations had recurrences available for analysis). It is important to note that the initial tumor resections of three of these tumors were not sequenced for SETD2, so it is uncertain if the changes were present at the time of initial presentation. However, one patient (\#13) had an IDH-wildtype anaplastic astrocytoma recur with progression to glioblastoma; the initial frontal lobe resection and the frontal lobe recurrence demonstrated the same mutational profile, including the same SETD2 missense mutation, whereas an intervening temporoparietal tumor resection demonstrated different mutations. EGFR p.G589 V and a copy number gain of EGFR were detected in both frontal lobe resections, and EGFR p.T263P and PTEN p.T366Hfs"50 were detected in the temporoparietal resection. In these recurrent/residual high grade gliomas with changes in SETD2, longer durations of follow-up were seen after initial presentation (mean of 79 and 42 months for $I D H$-mutant tumors and $52.5 \pm$ 12.0 months for $I D H$-wildtype tumors) than is common for high grade gliomas. Further studies are required to better elucidate how nonsense or frameshift mutations and missense mutations in SETD2 relate to prognosis.

Another question that requires further investigation is the difference between truncating mutations and missense SETD2 mutations. Prior Western blot studies found a decrease in levels of H3K36me3 for both truncating and missense mutations [6]. As a number of different missense mutations occurring throughout the SETD2 gene have been seen in CNS tumors, as well as in other tumor types, the role of individual missense mutations in tumorigenesis is unclear. One possibility is that a subset of missense mutations are passenger mutations that occur following glioma therapy. Three recurrent high grade gliomas (\#12, 14 and 16) in our subset had multiple missense mutations in SETD2. In these cases, a higher mutational burden was present following chemoradiation. It is likely that the missense SETD2 mutations seen at low VAF are not drivers of tumorigenesis in these tumors. In contrast, for the tumors with nonsense or frameshift mutations in SETD2, fewer concurrent PM were detected, and in one tumor (\#3), the PM in SETD2 was the only mutation detected on the NGS panel, at a VAF of $23 \%$.

\section{Conclusions}

In summary, these findings suggest that SETD2 mutations, although most common in high grade gliomas of the cerebral hemispheres, may be found in a variety of primary CNS tumors and locations. Immunohistochemistry shows a decrease in H3K36me3 in tumor with SETD2 mutations, implicating epigenetic pathways in tumor biology. Additional studies are needed to investigate the role of SETD2 mutations in tumorigenesis.

\section{Abbreviations}

CCRCC: Clear cell renal cell carcinoma; CHOP: Children's Hospital of Philadelphia; CNS: Central nervous system; CPD: Center for Personalized Diagnostics; DGD: Division of Genomic Diagnostics; H3K27me3: Trimethylation of the lysine 27 residue on Histone 3; H3K36ac: Acetylation of the lysine 36 residue on Histone 3; $\mathrm{H} 3 \mathrm{~K} 36 \mathrm{me} 3$ : Trimethylation of the lysine 36 residue on Histone 3; HOTAIR: HOX Transcript Antisense RNA; HUP: Hospital of the University of Pennsylvania; IHC: Immunohistochemistry; MM: Missense mutation; NGS: Next generation sequencing; SETD2: SET domain-containing 2; TCGA: The Cancer Genome Atlas; TM: Truncating mutation; VAF: Variant allele frequency

\section{Acknowledgements}

The authors thank Dr. John Wojcik for thoughtful review of this work and Daniel Martinez and the Children's Hospital of Philadelphia Pathology Core Laboratory for optimizing and performing immunohistochemistry.

\section{Funding}

Not applicable.

\section{Availability of data and materials}

The Cancer Genome Atlas (TCGA) dataset is available from cbioportal (http://www.cbioportal.org).

For any other data related to the University of Pennsylvania and Children's Hospital of Philadelphia cases, please contact the corresponding author (MPN).

Authors' contributions

ANV and MPN Conceived and designed the analysis; Collected the data; Contributed data; Performed the analysis; Wrote the paper. MS Contributed data and immunohistochemical staining. LFS, MML and JR Contributed data and assisted in analysis. All authors read and approved the final manuscript.

Ethics approval and consent to participate

This study was approved by an independent institutional review board at the Hospital of the University of Pennsylvania (HUP IRB 827290).

Consent for publication

Not applicable.

\section{Competing interests}

The authors declare that they have no competing interests.

\section{Publisher's Note}

Springer Nature remains neutral with regard to jurisdictional claims in published maps and institutional affiliations.

\section{Author details}

'Department of Pathology and Laboratory Medicine, Children's Hospital of Philadelphia, University of Pennsylvania Perelman School of Medicine, Philadelphia, PA, USA. ${ }^{2}$ Department of Pathology and Laboratory Medicine, University of Pennsylvania Perelman School of Medicine, Philadelphia, PA, USA. ${ }^{3}$ Hospital of the University of Pennsylvania, FO6.089 3400 Spruce St, Philadelphia, PA 19104, USA.

Received: 24 September 2018 Accepted: 21 October 2018

Published online: 12 November 2018

References

1. Brennan CW, Verhaak RG, McKenna A, Campos B, Noushmehr H, Salama SR, Zheng S, Chakravarty D, Sanborn JZ, Berman SH, Beroukhim R, Bernard B, Wu CJ, Genovese G, Shmulevich I, Barnholtz-Sloan J, Zou L, Vegesna R, Shukla SA, Ciriello G, Yung WK, Zhang W, Sougnez C, Mikkelsen T, Aldape K, Bigner DD, Van Meir EG, Prados M, Sloan A, Black KL, Eschbacher J, Finocchiaro G, Friedman W, Andrews DW, Guha A, lacocca M, O'Neill BP, Foltz G, Myers J, Weisenberger DJ, Penny R, Kucherlapati R, Perou CM, Hayes DN, Gibbs R, Marra M, Mills GB, Lander E, Spellman P, Wilson R, Sander C, Weinstein J, Meyerson M, Gabriel S, Laird PW, Haussler D, Getz G, Chin L, Network TR (2013) The somatic genomic landscape of glioblastoma. Cell 155:462-477. https://doi.org/10.1016/j.cell.2013.09.034

2. Butler JS, Koutelou E, Schibler AC, Dent SY (2012) Histone-modifying enzymes: regulators of developmental decisions and drivers of human disease. Epigenomics 4:163-177. https://doi.org/10.2217/epi.12.3 
3. Cancer Genome Atlas Research N (2008) Comprehensive genomic characterization defines human glioblastoma genes and core pathways. Nature 455:1061-1068. https://doi.org/10.1038/nature07385

4. Cerami E, Gao J, Dogrusoz U, Gross BE, Sumer SO, Aksoy BA, Jacobsen A, Byrne CJ, Heuer ML, Larsson E, Antipin Y, Reva B, Goldberg AP, Sander C, Schultz N (2012) The cBio cancer genomics portal: an open platform for exploring multidimensional cancer genomics data. Cancer Discov 2:401-404. https:/doi.org/10.1158/2159-8290.CD-12-0095

5. Duns $G$, van den Berg E, van Duivenbode I, Osinga J, Hollema H, Hofstra RM, Kok K (2010) Histone methyltransferase gene SETD2 is a novel tumor suppressor gene in clear cell renal cell carcinoma. Cancer Res 70:4287-4291. https://doi.org/10.1158/0008-5472.CAN-10-0120

6. Fontebasso AM, Schwartzentruber J, Khuong-Quang DA, Liu XY, Sturm D, Korshunov A, Jones DT, Witt H, Kool M, Albrecht S, Fleming A, Hadjadj D, Busche S, Lepage P, Montpetit A, Staffa A, Gerges N, Zakrzewska M, Zakrzewski K, Liberski PP, Hauser P, Garami M, Klekner A, Bognar L, Zadeh G, Faury D, Pfister SM, Jabado N, Majewski J (2013) Mutations in SETD2 and genes affecting histone H3K36 methylation target hemispheric high-grade gliomas. Acta Neuropathol 125:659-669. https:/doi.org/10.1007/s00401-013-1095-8

7. Gao J, Aksoy BA, Dogrusoz U, Dresdner G, Gross B, Sumer SO, Sun Y, Jacobsen A, Sinha R, Larsson E, Cerami E, Sander C, Schultz N (2013) Integrative analysis of complex cancer genomics and clinical profiles using the cBioPortal. Sci Signal 6:pl1. https://doi.org/10.1126/scisignal.2004088

8. Ho TH, Park IY, Zhao H, Tong P, Champion MD, Yan H, Monzon FA, Hoang A, Tamboli P, Parker AS, Joseph RW, Qiao W, Dykema K, Tannir NM, Castle EP, Nunez-Nateras R, Teh BT, Wang J, Walker CL, Hung MC, Jonasch E (2016) Highresolution profiling of histone $\mathrm{h} 3$ lysine 36 trimethylation in metastatic renal cell carcinoma. Oncogene 35:1565-1574. https://doi.org/10.1038/onc.2015.221

9. Hunter C, Smith R, Cahill DP, Stephens P, Stevens C, Teague J, Greenman C, Edkins S, Bignell G, Davies H, O'Meara S, Parker A, Avis T, Barthorpe S, Brackenbury L, Buck G, Butler A, Clements J, Cole J, Dicks E, Forbes S, Gorton M, Gray K, Halliday K, Harrison R, Hills K, Hinton J, Jenkinson A, Jones D, Kosmidou V, Laman R, Lugg R, Menzies A, Perry J, Petty R, Raine K, Richardson D, Shepherd R, Small A, Solomon H, Tofts C, Varian J, West S, Widaa S, Yates A, Easton DF, Riggins G, Roy JE, Levine KK, Mueller W, Batchelor TT, Louis DN, Stratton MR, Futreal PA, Wooster R (2006) A hypermutation phenotype and somatic MSH6 mutations in recurrent human malignant gliomas after alkylator chemotherapy. Cancer Res 66: 3987-3991. https://doi.org/10.1158/0008-5472.CAN-06-0127

10. Jones DT, Jager N, Kool M, Zichner T, Hutter B, Sultan M, Cho YJ, Pugh TJ, Hovestadt V, Stutz AM, Rausch T, Warnatz HJ, Ryzhova M, Bender S, Sturm D, Pleier S, Cin H, Pfaff E, Sieber L, Wittmann A, Remke M, Witt H, Hutter S, Tzaridis T, Weischenfeldt J, Raeder B, Avci M, Amstislavskiy V, Zapatka M, Weber UD, Wang Q, Lasitschka B, Bartholomae CC, Schmidt M, von Kalle C, Ast V, Lawerenz C, Eils J, Kabbe R, Benes V, van Sluis P, Koster J, Volckmann R, Shih D, Betts MJ, Russell RB, Coco S, Tonini GP, Schuller U, Hans V, Graf N, Kim YJ, Monoranu C, Roggendorf W, Unterberg A, Herold-Mende C, Milde T, Kulozik AE, von Deimling A, Witt O, Maass E, Rossler J, Ebinger M, Schuhmann MU, Fruhwald MC, Hasselblatt M, Jabado N, Rutkowski S, von Bueren AO, Williamson D, Clifford SC, McCabe MG, Collins VP, Wolf S, Wiemann S, Lehrach H, Brors B, Scheurlen W, Felsberg J, Reifenberger G, Northcott PA, Taylor MD, Meyerson M, Pomeroy SL, Yaspo ML, Korbel JO, Korshunov A, Eils R, Pfister SM, Lichter P (2012) Dissecting the genomic complexity underlying medulloblastoma. Nature 488:100-105. https://doi. org/10.1038/nature 1284

11. Ia Rosa AH, Acker M, Swain S, Manoharan M (2015) The role of epigenetics in kidney malignancies. Cent Eur J Urol 68:157-164. https://doi.org/10.5173/ ceju.2015.453

12. Lek M, Karczewski KJ, Minikel EV, Samocha KE, Banks E, Fennell T, O'DonnellLuria AH, Ware JS, Hill AJ, Cummings BB, Tukiainen T, Birnbaum DP, Kosmicki JA, Duncan LE, Estrada K, Zhao F, Zou J, Pierce-Hoffman E, Berghout J, Cooper DN, Deflaux N, DePristo M, Do R, Flannick J, Fromer M, Gauthier L, Goldstein J, Gupta N, Howrigan D, Kiezun A, Kurki MI, Moonshine AL, Natarajan P, Orozco L, Peloso GM, Poplin R, Rivas MA, Ruano-Rubio V, Rose SA, Ruderfer DM, Shakir K, Stenson PD, Stevens C, Thomas BP, Tiao G, Tusie-Luna MT, Weisburd B, Won HH, Yu D, Altshuler DM, Ardissino D, Boehnke M, Danesh J, Donnelly S, Elosua R, Florez JC, Gabriel SB, Getz G, Glatt SJ, Hultman CM, Kathiresan S, Laakso M, McCarroll S, McCarthy MI, McGovern D, McPherson R, Neale BM, Palotie A, Purcell SM, Saleheen D, Scharf JM, Sklar P, Sullivan PF, Tuomilehto J, Tsuang MT, Watkins HC, Wilson JG, Daly MJ, MacArthur DG, Exome Aggregation C
(2016) Analysis of protein-coding genetic variation in 60,706 humans. Nature 536:285-291. https://doi.org/10.1038/nature19057

13. Li F, Mao G, Tong D, Huang J, Gu L, Yang W, Li GM (2013) The histone mark H3K36me3 regulates human DNA mismatch repair through its interaction with MutSalpha. Cell 153:590-600. https://doi.org/10.1016/j. cell.2013.03.025

14. Li J, Duns G, Westers H, Sijmons R, van den Berg A, Kok K (2016) SETD2: an epigenetic modifier with tumor suppressor functionality. Oncotarget 7 : 50719-50734. https://doi.org/10.18632/oncotarget.9368

15. Li MM, Datto M, Duncavage EJ, Kulkarni S, Lindeman NI, Roy S, Tsimberidou AM, Vnencak-Jones CL, Wolff DJ, Younes A, Nikiforova MN (2017) Standards and guidelines for the interpretation and reporting of sequence variants in Cancer: A Joint Consensus Recommendation of the Association for Molecular Pathology, American Society of Clinical Oncology, and College of American Pathologists. J Mol Diagn 19:4-23. https://doi.org/10.1016/j. jmoldx.2016.10.002

16. Liu W, Fu Q, An H, Chang Y, Zhang W, Zhu Y, Xu L, Xu J (2015) Decreased expression of SETD2 predicts unfavorable prognosis in patients with nonmetastatic clear-cell renal cell carcinoma. Medicine (Baltimore) 94:e2004. https://doi.org/10.1097/MD.0000000000002004

17. Long W, Yi Y, Chen S, Cao Q, Zhao W, Liu Q (2017) Potential new therapies for pediatric diffuse intrinsic pontine glioma. Front Pharmacol 8:495. https://doi.org/10.3389/fphar.2017.00495

18. Louis DN, Perry A, Reifenberger G, von Deimling A, Figarella-Branger D, Cavenee WK, Ohgaki H, Wiestler OD, Kleihues P, Ellison DW (2016) The 2016 World Health Organization classification of tumors of the central nervous system: a summary. Acta Neuropathol 131:803-820. https://doi.org/10.1007/ s00401-016-1545-1

19. Lu C, Jain SU, Hoelper D, Bechet D, Molden RC, Ran L, Murphy D, Venneti S, Hameed M, Pawel BR, Wunder JS, Dickson BC, Lundgren SM, Jani KS, De Jay N, Papillon-Cavanagh S, Andrulis IL, Sawyer SL, Grynspan D, Turcotte RE, Nadaf J, Fahiminiyah S, Muir TW, Majewski J, Thompson CB, Chi P, Garcia BA, Allis CD, Jabado N, Lewis PW (2016) Histone H3K36 mutations promote sarcomagenesis through altered histone methylation landscape. Science 352:844-849. https://doi.org/10.1126/science.aac7272

20. Marmorstein R, Trievel RC (2009) Histone modifying enzymes: structures, mechanisms, and specificities. Biochim Biophys Acta 1789:58-68. https://doi. org/10.1016/j.bbagrm.2008.07.009

21. Pai CC, Deegan RS, Subramanian L, Gal C, Sarkar S, Blaikley EJ, Walker C, Hulme L, Bernhard E, Codlin S, Bahler J, Allshire R, Whitehall S, Humphrey TC (2014) A histone H3K36 chromatin switch coordinates DNA doublestrand break repair pathway choice. Nat Commun 5:4091. https://doi.org/10. 1038/ncomms5091

22. Suzuki H, Aoki K, Chiba K, Sato Y, Shiozawa Y, Shiraishi Y, Shimamura T, Niida A, Motomura K, Ohka F, Yamamoto T, Tanahashi K, Ranjit M, Wakabayashi T, Yoshizato T, Kataoka K, Yoshida K, Nagata Y, Sato-Otsubo A, Tanaka H, Sanada M, Kondo Y, Nakamura H, Mizoguchi M, Abe T, Muragaki Y, Watanabe R, Ito I, Miyano S, Natsume A, Ogawa S (2015) Mutational landscape and clonal architecture in grade II and III gliomas. Nat Genet 47: 458-468. https://doi.org/10.1038/ng.3273

23. Toh TB, Lim JJ, Chow EK (2017) Epigenetics in cancer stem cells. Mol Cancer 16:29. https://doi.org/10.1186/s12943-017-0596-9

24. Walter DM, Venancio OS, Buza EL, Tobias JW, Deshpande C, Gudiel AA, KimKiselak C, Cicchini M, Yates TJ, Feldser DM (2017) Systematic in vivo inactivation of chromatin-regulating enzymes identifies Setd2 as a potent tumor suppressor in lung adenocarcinoma. Cancer Res 77:1719-1729. https://doi.org/10.1158/0008-5472.CAN-16-2159

25. Wang L, Zehir A, Nafa K, Zhou N, Berger MF, Casanova J, Sadowska J, Lu C, Allis CD, Gounder M, Chandhanayingyong C, Ladanyi M, Boland PJ, Hameed M (2016) Genomic aberrations frequently alter chromatin regulatory genes in chordoma. Genes Chromosomes Cancer 55:591-600. https://doi.org/10. 1002/gcc.22362

26. Wilting RH, Dannenberg JH (2012) Epigenetic mechanisms in tumorigenesis, tumor cell heterogeneity and drug resistance. Drug Resist Updat 15:21-38. https://doi.org/10.1016/j.drup.2012.01.008

27. Wu G, Diaz AK, Paugh BS, Rankin SL, Ju B, Li Y, Zhu X, Qu C, Chen X, Zhang J, Easton J, Edmonson M, Ma X, Lu C, Nagahawatte P, Hedlund E, Rusch M, Pounds $\mathrm{S}$, Lin T, Onar-Thomas A, Huether R, Kriwacki R, Parker M, Gupta P, Becksfort J, Wei L, Mulder HL, Boggs K, Vadodaria B, Yergeau D, Russell JC, Ochoa K, Fulton RS, Fulton LL, Jones C, Boop FA, Broniscer A, Wetmore C, Gajjar A, Ding L, Mardis ER, Wilson RK, Taylor MR, Downing JR, Ellison DW, 
Zhang J, Baker SJ (2014) The genomic landscape of diffuse intrinsic pontine glioma and pediatric non-brainstem high-grade glioma. Nat Genet 46:444-450. https://doi.org/10.1038/ng.2938

28. Xie P, Tian C, An L, Nie J, Lu K, Xing G, Zhang L, He F (2008) Histone

methyltransferase protein SETD2 interacts with $\mathrm{p} 53$ and selectively regulates its downstream genes. Cell Signal 20:1671-1678. https://doi.org/10.1016/j. cellsig.2008.05.012

Ready to submit your research? Choose BMC and benefit from:

- fast, convenient online submission

- thorough peer review by experienced researchers in your field

- rapid publication on acceptance

- support for research data, including large and complex data types

- gold Open Access which fosters wider collaboration and increased citations

- maximum visibility for your research: over $100 \mathrm{M}$ website views per year

At BMC, research is always in progress.

Learn more biomedcentral.com/submissions 